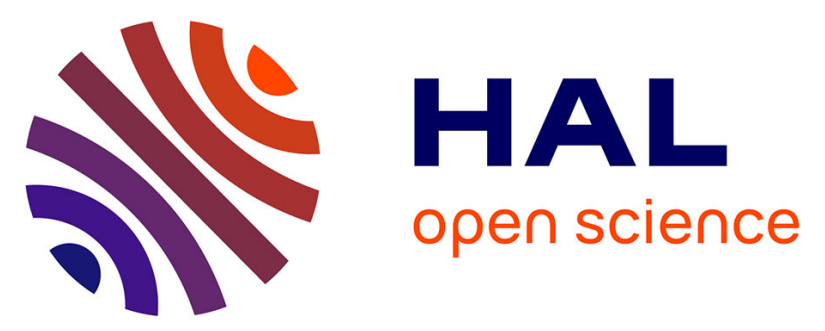

\title{
DROSERA: A DROne Simulation Environment for Risk Assessment
}

Nicolas Raballand, Sylvain Bertrand, Stéphanie Lala, Baptiste Levasseur

\section{To cite this version:}

Nicolas Raballand, Sylvain Bertrand, Stéphanie Lala, Baptiste Levasseur. DROSERA: A DROne Simulation Environment for Risk Assessment. Proceedings of the 31st European Safety and Reliability Conference, Sep 2021, Angers, France. pp.354-361, 10.3850/978-981-18-2016-8_236-cd . hal03420529

\section{HAL Id: hal-03420529 \\ https://hal.science/hal-03420529}

Submitted on 9 Nov 2021

HAL is a multi-disciplinary open access archive for the deposit and dissemination of scientific research documents, whether they are published or not. The documents may come from teaching and research institutions in France or abroad, or from public or private research centers.
L'archive ouverte pluridisciplinaire HAL, est destinée au dépôt et à la diffusion de documents scientifiques de niveau recherche, publiés ou non, émanant des établissements d'enseignement et de recherche français ou étrangers, des laboratoires publics ou privés. 


\title{
DROSERA: a DROne Simulation Environment for Risk Assessment
}

\author{
Nicolas Raballand, Sylvain Bertrand, Stéphanie Lala, Baptiste Levasseur \\ Université Paris-Saclay, ONERA, Traitement de l'Information et Systèmes, Palaiseau, France. \\ E-mail: nicolas.raballand@onera.fr, sylvain.bertrand@onera.fr, stephanie.lala@onera.fr, \\ baptiste.levasseur@onera.fr
}

\begin{abstract}
The use of Unmanned Aerial Vehicles (UAVs), or drones, appears to be a very effective means for aerial inspection of outdoor infrastructures over large areas and data collection. For these missions to be authorized, drone operators must ensure that flight paths respect a given level of safety with regard to third parties such as population, transportation networks, critical infrastructures, etc. This paper presents a tool (DROSERA) for probabilistic risk assessment of fixed-wing UAV missions. It aims to calculate the probability of getting casualties for people at ground or accidents on transportation networks due to loss of control of an UAV. This tool integrates several models for such probability calculations, from the literature or developed by the authors. New models are presented here such as specific flight termination strategies for risk mitigation (terminal spiral), time of day sensitivity (people and traffic), effect of wind conditions. A synthesis of these models, their integration into the DROSERA tool, required inputs and calculated outputs are presented and illustrated on an example of UAV mission in a semi-urban scenario.
\end{abstract}

Keywords: Probabilistic risk assessment, Unmanned Aerial Vehicles, Simulation framework.

\section{Introduction}

The continuous progress in the areas of Unmanned Aerial Vehicles (UAVs) and associated embedded sensors lead the owner of large infrastructure networks to study more and more closely the deployment of drones to monitor these facilities. It seems to be an efficient way to improve their ability to get an overview of their infrastructures and if necessary to trigger the expected maintenance actions.

Nevertheless, some external constraints have to be taken into account before taking advantage of these new means. As these infrastructure networks often provide services to the population at national level, they extend to different areas, which can be rural as well as densely populated. One can easily figure out that surrounding the population with aerial vehicles performing inspection missions raises some issues and must be tackled with precaution. This is the reason why drone operators have to ensure a high level of safety concerning the aircraft themselves but also regarding the missions planned. While the goal of the first aspect is to prevent the failures on the UAVs, the second aspect aims to limit the damages to third parties when such failures occur, by considering the whole trajectory of a flight along with its environment.

Probabilistic Risk Assessment (PRA) approaches have received a huge interest in the UAV community as a quantitative way for risk assessment, at a mission level, in complement to other more qualitative approaches (Washington et al.
(2017)). Mainly focused on ground risk for people of inhabited areas, works in the literature have developed models to contribute to the probabilistic computation of the occurrence (see e.g. Wu and Clothier (2012); Melnyk et al. (2014); Bertrand et al. (2017)), and severity (see e.g. Dalamagkidis et al. (2008); Rattanagraikanakorn et al. (2020)) of an accident caused by the collision between an UAV falling at ground and a person. More recently, the authors have developed and proposed some specific models to evaluate the risk of causing an accident on road networks (or more generally on ground transportation networks) due to the fall of an UAV (Bertrand et al. (2018)). All these models can be used for computation of risk indicators (namely probabilities) associated with any flight, involving the presence of people and other human activities in the surrounding area that might be impacted. This is of a major help for mission preparation and for the process of getting flight authorizations. Nevertheless, one can easily understand that this work can be time-consuming when applying to large facilities as routine activities. It is all the more true that this stage implies to take into account many context dependent information such as UAV characteristics, ground relief, population, infrastructures, weather conditions, time of the day, etc.

Development of a tool that integrates all the models and required data for risk assessment is of interest for various stakeholders: drone operators to prepare flights in compliance with the rules, authority to check the flight authorization requests, UAV manufacturers to assess mitigation means for 
risk or procedures like Flight Termination Systems (FTS), infrastructure managers to identify parts of their facilities eligible for aerial inspection and to manage corresponding assets, etc. This paper presents such a tool, named DROSERA, for probabilistic risk assessment of fixed-wing UAV missions wrt people of inhabited areas and ground transportation networks. New models are also presented in this paper to account for some specific FTS for risk mitigation (terminal spiral), sensitivity to the hour of the day (people and traffic), effect of wind conditions.

The next section describes the main steps of the PRA method and introduces the different types of models and inputs required. Section 3 provides a focus on some models for which specific developments are proposed by the authors. These models are included in the DROSERA tool which structure is presented in Section 4, along with a description of the generated outputs. A case study of a long range flight mission over a semi-urban area is then proposed in Section 5 for illustration. Conclusions are given in the last section of the paper.

\section{Probabilistic Risk Assessment Method}

Risk assessment can be done by using qualitative or quantitative methods. Quantitative methods are more suited to the problem of interest since comparison to some expected levels of safety is interesting. For instance, one should be able to assess that the risk induced by the use of an UAV does not exceed the one of manned aviation, usually defined in numbers of fatalities per flight hour.

To be able to perform quantitative risk assessment, models should be developed that represent the system and its environment and allow to compute quantitative indicators of the risk. These models should be able to cope with uncertainties due to modeling errors, possible variations of environmental conditions, probabilistic data available on some components, etc. For these reasons, model-based Probabilistic Risk Assessment has received a great attention from the UAV community to develop methods and required models in order to compute the probability of getting fatalities to third parties due to the use of an UAV.

To compute such a probability the risk under consideration is decomposed into a sequence of several elementary feared hazards for which a specific probability can be calculated. Following the approach introduced in Clothier et al. (2007), the probability to get casualties to third parties is computed as

$$
\begin{array}{r}
\operatorname{Pr}\{\text { casualty }\}=\operatorname{Pr}\{\text { loss }\} . \operatorname{Pr}\{\text { impact } \mid \text { loss }\} \\
. \operatorname{Pr}\{\text { collision } \mid \text { impact } \cap \text { loss }\} \\
. \operatorname{Pr}\{\text { damage } \mid \text { collision } \cap \text { impact } \cap \text { loss }\}
\end{array}
$$

The first term is related to the UAV itself and corresponds to the probability to get a failure leading to a loss of control of the vehicle. In this work, engine failure is considered as the most probable event leading to a loss of control on the trajectory according to returns on experience from manufacturers and literature. It can also be computed for example using Model Based Safety Assessment methods considering the whole physical and software architectures of the UAV (Bieber et al. (2018), Delmas et al. (2019)). The second term in (1) refers to the probability of impact at ground and should be evaluated for all possible impact locations of the UAV. Note that this impact area at ground depends on the descent dynamics of the vehicle to ground and can be influenced by external parameters such as wind. The third term in (1) is the probability of the drone to collide with a third party (people, car, etc.). It is directly related to the presence of third parties in the ground impact area computed in the previous stage. Finally, the last term in (1) is an expression of the severity of the collision. Roughly speaking, it is the probability of a lethal impact to happen. More information on the computation of these four terms can be found in Wu and Clothier (2012), Melnyk et al. (2014), Washington et al. (2017), or Bertrand et al. (2017).

Such a decomposition as in (1) allows developing and iteratively refining models for each term computation independently from the others, according to availability of required data, e.g. databases related to third parties' presence (population density, road traffic) or data coming from the operators and manufacturers' returns on experience. Expression (1) also highlights the fact that the assessment depends on two main classes of models and parameters: those related to the aerial vehicle itself and those related to its environment. Specific models developed with regard to these two classes are presented in the next section.

\section{Specific Models Developed for Probabilistic Risk Assessment}

This section provides more technical details on some of the models used for the evaluation of (1) and for which specific developments have been carried out by the authors.

\subsection{UAV Ground Impact Model}

A particular attention has been paid to the development of accurate models enabling to compute ground impact footprints and associated probability maps for the UAV, that will be used in the computation of $\operatorname{Pr}\{$ impact|loss $\}$. In addition to the type of failure and resulting uncontrolled descent to the ground, these probability maps strongly depend on the characteristics of the UAV, possibly leading to huge differences from one vehicle to 

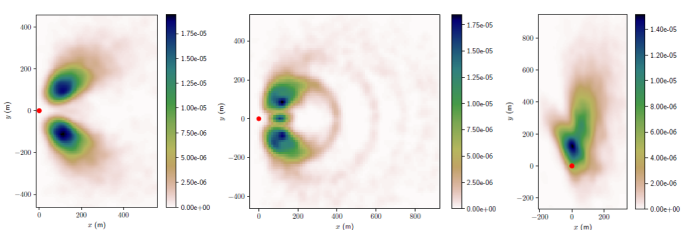

Fig. 1. Ground impact probability maps: no wind $h_{0}=150 \mathrm{~m}$, no wind $h_{0}=50 \mathrm{~m}$, wind $h_{0}=150 \mathrm{~m}$

another. A first model considered in the literature (Clothier et al. (2007)) is based on the assumption of a gliding descent of the vehicle. It has been used by the authors in Bertrand et al. (2017) as a first basis, assuming that the locations of the ground impact points follow a normal distribution which mean is halfway between the horizontal position of the UAV at the instant of failure and the touchdown and which covariance is intuited from the maximum gliding distance depending on the lift to drag ratio of the drone. This model is easy to use in terms of computation time for risk assessment. Although simplistic, it can be tuned to fit some bounding box dimensions of crash footprints provided by some drone manufacturers, and can easily be modified to account for risk mitigation procedures (e.g. parachute or flight termination procedure such as spiral controlled descent).

Nevertheless this model does not account for flight dynamics of the vehicle during the descent to ground, nor possible effects of other actuators (e.g. deflection of control surfaces), and may lead to large errors in the values of the computed impact probabilities. This is why a methodology has been developed by the authors in Levasseur et al. (2019) to generate accurate ground impact probability maps, based on Monte Carlo simulations of descent trajectories using a dynamical model with flight mechanics of the UAV that accounts for aerodynamics, effect of actuators, and influence of wind. Examples of impact probability maps obtained by this approach are presented in Fig. 1. Several modes can be seen in the probability distributions which can hardly be modelled representatively enough by a normal distribution. Effect of wind is also clearly visible, which is very important for long range outdoor missions where wind conditions can be non negligible and may be very changing during the flight. The main problem of this approach is that Monte Carlo simulations are time prohibitive to be used in an integrated tool such as the one presented in this paper (DROSERA), which aims at evaluating the risk at each point of the flight trajectory, for long range missions, and possibly for a huge number of missions when considering inspection of infrastructure at a country scale Bertrand et al. (2019). Therefore, a full data basis of ground impact probability maps has been generated "offline", considering a large set of possible values for parame- ters of influence (altitude, speed, wind direction and speed, etc.). For each map, a probability distribution based on Gaussian mixtures has been fitted. Parameters of all these distributions are stored in another data basis which is used by the DROSERA tool. Probability computation is indeed achieved by choosing parameters of a given distribution or interpolating between several in the data basis, depending on all the different successive flight conditions encountered along the simulated trajectory. This solution enables fast risk computation by the DROSERA tool, while preserving accuracy compared to a simplistic model. Note that, development of surrogate models based on neural networks (Levasseur et al. (2020)) are also under investigation for computation time reduction.

\subsection{Third Parties}

As presented in Section 2, the risk to be evaluated concerns third-party casualties due to collisions with an UAV impacting at ground. The most vulnerable third-party entities that have to be considered are persons, when being outside, i.e. not sheltered by any building. In this case, the damage caused by the collision is direct and is accounted for in the evaluation by the third (occurrence of collision) and fourth (severity of collision) terms of equation (1). Indirect damages to people must also be considered, e.g. induced by a traffic accident if the UAV crashes on a road. Although the first type of "direct" damages has been considered in the literature, the second one regarding traffic accidents has been specifically modelled by the authors in Bertrand et al. (2018) and is implemented in the DROSERA tool as well.

The main difficulty in modeling the presence of third parties (people, vehicles on road networks) is to get a realistic picture of human activities from two points of view: geographically, in the area of interest corresponding to the flight trajectory (more specifically inside the ground impact footprint along the flight trajectory), and temporally, since flights can be performed at different hours of the day. In the future, with drones of long endurance one should also be able to address flight of possibly several hours and to cope with the fact that people's habits can vary from hour to hour. An accurate model of third partie's presence must therefore rely on both static aspects (locations of inhabited areas or road networks) and dynamical ones (time variations of peoples' presence outside and of road traffic during the day).

Regarding the static aspect of these third parties, the developed method relies on multiple databases coming from open source information including terrain elevation, population density and road networks. Resolution of the databases is of a paramount importance, as results of risk evaluation will directly depend on it. Regarding popula- 
tion density, the need is to reflect a realistic risk of vulnerability for persons (e.g. urban areas may count a lot of people but presence of buildings also offers protection). The realism is therefore enhanced using land coverage information that helps in showing that the presence of buildings can protect people from direct collision with high kinetic energy. As this sheltering factor is not available directly the assumption is made that for urban and semi urban areas the surface at ground that may be impacted and over which damage to people may result is diminished by the average surface of buildings in these areas. The population density is therefore multiplied by a corrective factor to account for this sheltering possibility.

Considering the dynamical aspect desired in models of third parties' presence, the mean agenda of the population should be considered. Indeed, the presence of population in a specific area fluctuates during the day (and the night). Starting from a survey by the French National Institute of Statistics and Economic Studies (INSEE (2012)) showing that outdoor activities represent less than $20 \%$ of the daily schedule, another corrective factor is applied to the population density to take into account the fact that persons are mostly inside buildings during the night and are likely to be outside just before and after business hours. Sensitivity to the hour of the day is therefore accounted for in the population density that is used in the DROSERA tool, as shown on the example of Fig. 2 left. In this example, the red and blue lines respectively represent the population density data without and with correction accounting for people's habits and hour of the day.

Regarding road traffic, data from open traffic surveys have been compiled to produce mean average daily traffic values as well as vehicle speeds representative of different types of roads (see Bertrand et al. (2018) for more details). Then a similar approach has been taken to model the influence of the day hour on traffic density and speed, using daily car traffic trends from open sources. Fig. 2 right presents an example of the number of vehicles on a road section wrt the hour of the day. Peak hours are clearly visible. Note that another improvement would need to be conducted to involve habits during the week, to make a difference between working days and weekends, and also during the year since behaviors change with seasons (e.g. periods of holidays). All these models have been implemented in the DROSERA tool, which is described in the next section.

\section{DROSERA tool for Probabilistic Risk Assessment (PRA)}

Given the multiplicity of data involved in risk assessment of an UAV mission, it is important to dispose of a tool that integrates all these data and models and implements the method for Probabilis-
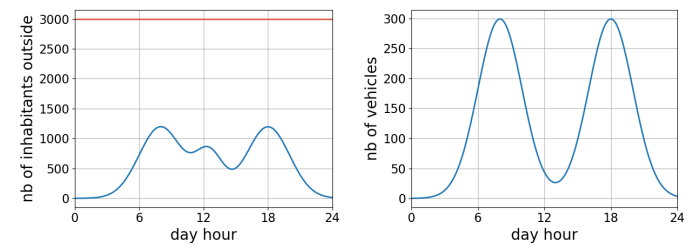

Fig. 2. Distribution of outdoor population (left) and distribution of road traffic (right) wrt day hour.

tic Risk Assessment as described in Section 2

The structure of the DROSERA tool is presented in Fig. 3 It is composed of three main stages that respectively concerns:

- the inputs required for risk assessment of the mission: data and models related to the UAV, its trajectory and its environment.

- the risk computation process that implements the PRA method for the different classes of third parties considered (people and roads).

- the post processing of the results (probabilities) to generate other indexes of interest for risk analysis and decision making.

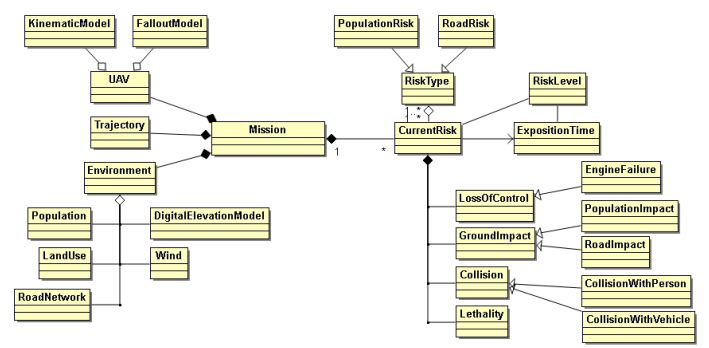

Fig. 3. Global architecture of the DROSERA tool.

The modular structure of the software enables progressive and agile improvements of some modules (e.g. new type of failure or mitigation procedure regarding the UAV and descent models), addition of new parameters, definition of new indexes for interpretation of the results, other types of risks to be considered in the evaluation, etc. Software modules are implemented in $\mathrm{C}++$ (simulation and risk computation) or Python (pre or post processing). DROSERA can be used either with its HMI or in a batch mode with fast computation, allowing multi runs for Monte Carlo simulations or risk analysis over large scenarios (e.g. French railways network as in Bertrand et al. (2019)).

\subsection{Inputs of DROSERA}

One can distinguish two kinds of inputs: those that can be chosen by the user for each mission from the main HMI of DROSERA (see Fig. 4), and 
those that rarely change and are "hidden" in the software.

The UAV characteristics are stored into a file depending on the simulation framework core; it gathers all the static and kinematic information used by the models, such as mass, dimensions, lift over drag ratio, probability of failure of components (main engine in this case), etc. Other parameters such as aerodynamic coefficients are not required but are used in the preliminary stage of generation of the data basis that will be used by DROSERA for computation of the ground impact probabilities (see Section 3.1).

Most of these parameters do not change once the user has decided of the operational configuration of the UAV. The HMI of DROSERA allows selecting among predefined UAV "profiles" and with or without means of mitigation when available on-board. Evaluation of the influence of mitigation means on the risk was indeed one purpose that led to the method definition.

The operator defines the flight trajectory that will be followed by the UAV according to the mission objectives and flight regulations. For use by DROSERA, the trajectory has to be defined as a succession of (latitude, longitude) with corresponding height above ground level. These data can be defined in a KML file which is easy to build and visualize using standard open tools already used by drone operators for flight preparation and mission planning. The user has also to specify in the DROSERA HMI the cruise speed and the takeoff time for the mission. Speed along the flight trajectory can be considered as constant or timevarying if required for the needs of the mission.

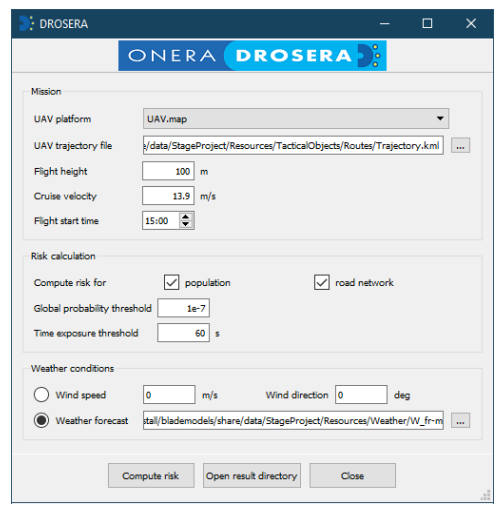

Fig. 4. HMI of the DROSERA tool.

DROSERA uses several types of environmental data. Some are closely linked to the chosen trajectory and need to be modified for each simulation, e.g. weather conditions (using forecasts in the standard GRIB2 format), some other are considered as long-term valid and are hidden to the user, e.g. geographical databases containing ground characteristics (digital elevation model, land coverage) and presence of third parties (population density, road networks). These data are discretized into georeferenced raster tiled files or vector files, according to the nature of the third parties they represent.

\subsection{Risk computation}

The risk is computed during the simulation run with respect to the third parties selected by the user. Population and/or road networks can be chosen for risk evaluation. Models for risk assessment wrt other types of third parties are currently under development and will be added to DROSERA.

The simulation of the mission consists in calculating step by step the successive positions of the UAV. For each step, DROSERA computes the four terms of the global risk equation (1). Among them, $\operatorname{Pr}\{$ impact|loss $\}$ and $\operatorname{Pr}\{$ collision $\mid$ impact $\cap$ loss $\}$ need the evaluation of the ground impact footprint: to apply the probabilistic distribution over the area of interest for the first one; to account for presence of third parties in this area for the second one. They are computed for each cell of the corresponding impact footprint (number and size of cells depending on the discretization for the third parties under consideration). These probabilities are associated to the current simulation time and stored as instantaneous values and the global risk probability is computed by multiplying them according to (1). In addition to these instantaneous values, the maximum values of the probabilities, for all instants, are stored for each cell at ground in any possible impact footprint (see Bertrand et al. (2017) for definition). Finally exposition time to the risk is also computed as the time duration each cell at ground belongs to a possible impact footprint during the whole mission (see also Bertrand et al. (2017) for definition).

\subsection{Outputs}

One can distinguish three categories of outputs, each with various goals. During the simulation first, the results of the current step in terms of probabilities and exposition time are stored into tables in order to create graphical files to be displayed on globe viewer, with the objective to locate areas or parts of trajectories requiring attention. Depending on the considered third parties, DROSERA chooses the suitable format to represent the associated risk. For population the input data corresponds to a grid based on a geographical database stored as tiled raster files (GeoTIFF), the output file keeps the same format. For road networks, the input data has the form of a vector file (ShapeFile), to facilitate further exploitation a similar format is used (KML).

However, probabilities and exposition time maps and charts may be complex for the user to interpret quickly. For this reason, more readable and 

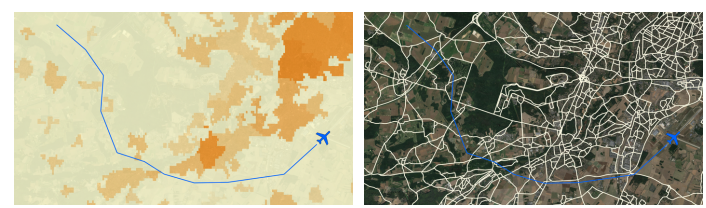

Fig. 5. Trajectory of the UAV, population density (left) and road network (right) in the area of the case study.

synthetic risk classes have been developed considering thresholds that can be chosen to reflect requirements on expected level of safety by flight authorities. This leads to building geo-referenced files similar to the previous ones but limited to three colors only (for high, medium and low risk).

A final configurable post-processing stage introduces operational and deployment constraints that may be used to identify interesting flight segments of a predefined trajectory or even to compare entire flights with each other. Thus, one can create charts with reference to the course of the mission, like bar graphs for feasibility (see Fig. 14), some other dedicated to get a global picture of a trajectory (see Fig. 13. By extension this approach may help in the prioritisation of a fleet deployment when large infrastructures are considered. Finally, the outputs are gathered into summary sheets thanks to configurable Python scripts, as a single point of entry for all the indicators.

\section{Case Study}

A case study was performed on a trajectory representative of a long-range and low altitude operation in a semi-urban area. The results presented hereafter show the risk indicators computed by the DROSERA tool for a $20 \mathrm{~km}$ trajectory where the UAV crosses diversely populated areas and roads. Flight trajectory of the UAV as well as the population density and road network corresponding to the case study are presented in Fig. 5

The global risk probability, as defined from (1), is computed over the flight and gives a representation as a map (Fig. 6left). If one observes that the flight trajectory has been planned to avoid as much as possible populated areas, some of them are nevertheless found in the ground impact footprint leading to "high" risk probabilities (see red areas in Fig. 6 left). With a probability threshold of $10 \mathrm{E}-7$ in the definition of risk classes, these areas appear with a "high risk" level (red color on Fig.6 right) if also associated with a high risk exposition time (i.e. exceeding both thresholds used for classification). Areas with only probability or exposition time greater than threshold appear in yellow ("medium risk") and others in green ("low risk"). Maps on Fig. 6 have been obtained using the simplified ground impact model which does not account for flight mechanics (as discussed in Section 3.1). When the same trajectory is evaluated
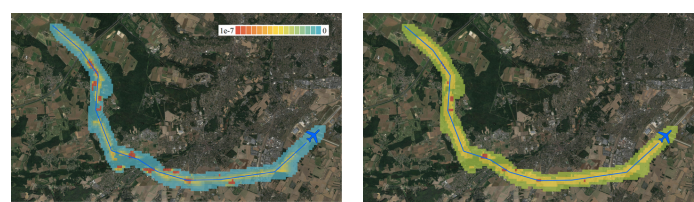

Fig. 6. Risk probability map (left) and risk level map (right) with the simplified ground impact model (see Section 3.1 .

under the assumption that the UAV is equipped with a FTS, the effectiveness of such equipment can be evaluated. By limiting the surface of the ground impact footprint and the kinetic energy at impact, the high risk areas disappear from the level risk maps leading to more areas tagged as 'low risk' with a FTS imposing a descent in a controlled spiral (cf. Fig. 7] left) and even more with a parachute (cf. Fig. 7 right, and Bertrand et al. (2017) for associated model). As mentioned in
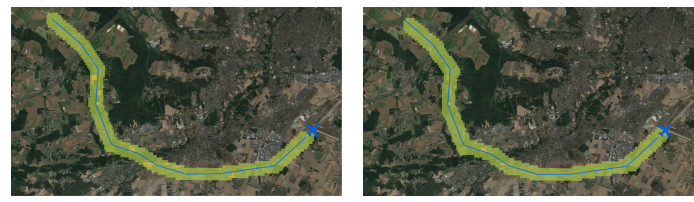

Fig. 7. Risk level maps for population computed with FTS for risk mitigation: spiral (left) / parachute (right).

Section 3.1, this simplified ground impact model does not account for flight dynamics nor effect of actuators and may lead to underestimation of the risk. To illustrate this point, the improved model based on the data basis of ground impact probability maps generated using a flight mechanics model (see Section 3.1) has been used to produce the risk level map of Fig. 8 left. Compared with Fig. 6 right one can see that the ground impact footprint is larger with the improved model accounting for the effects caused by the positions of the flaps at the instant of engine failure. When also accounting for wind in the computation, one can see some geographical offset in the risk map hence eventually encompassing other inhabited areas as shown on Fig. 8 right.

As explained in Section 3, the risk assessment methodology is similar whatever the third parties concerned. It is therefore applied to the overflown roads in the same manner as for the population. Fig. 9 presents the risk level maps computed wrt roads. Sections of roads in red are the one tagged as "high risk", the yellow as "medium risk", and the green as "low risk". High risk sections correspond to roads with a lot of traffic and would require special attention by the drone operator.

The result of the risk evaluation strongly depends on the time chosen for take-off. Fig. 10 

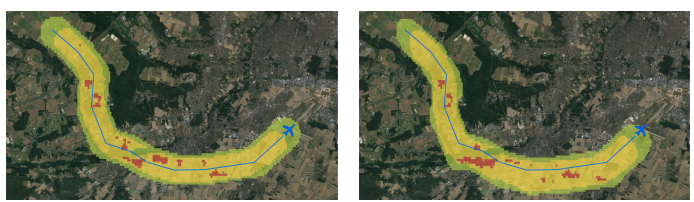

Fig. 8. Risk level maps wrt population : without wind (left) / with a wind of $10 \mathrm{~m} / \mathrm{s}$ coming from North (right).
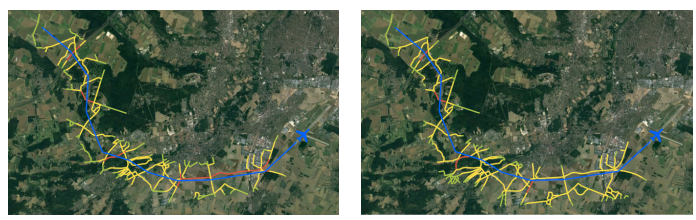

Fig. 9. Risk level maps wrt road network: without wind (left) / with a wind of $10 \mathrm{~m} / \mathrm{s}$ coming from North (right).

points out the discrepancy between a day flight at rush hour $(6 \mathrm{pm})$ on the left and a night flight (1am) on the right. All the high risk areas fade when people are mainly under the shelter of buildings during night. For the drone operator, geographical maps are of a major interest to visualize risks wrt third parties induced by the UAV mission and quickly identify areas that require to be closely studied during mission preparation. Nevertheless, these maps can be completed by complementary charts to help decision-making and understanding. DROSERA tool provides additional charts, such as the ones of Fig. 11 and Fig. 12, for time evolution of each term of equation (1) and for the resulting risk probability, or Fig. 13 for risk classification.

Once again, the classification into risk levels allows to take into account the regulatory thresholds and leads to the creation of three-color charts showing the evolution of the risk level as the flight progresses as shown on the top of Fig. 14 An alternative and more intuitive representation is built from these risk repartitions in the form of bar graphs (see bottom of Fig. 14) to classify and identify parts of the flight trajectory that should be considered too risky, and other parts for which the risk level could be considered as acceptable and operational flight would be possible. Operational constraints (e.g. deployment costs) are accounted
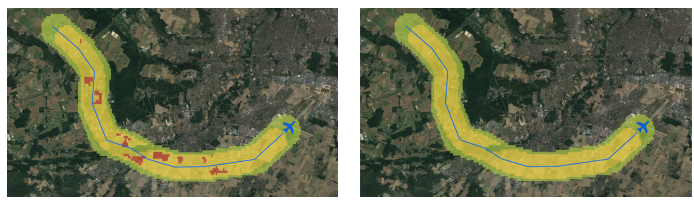

Fig. 10. Risk level map wrt population : with take-off at $6 \mathrm{pm}$ (left) and 1 am (right).

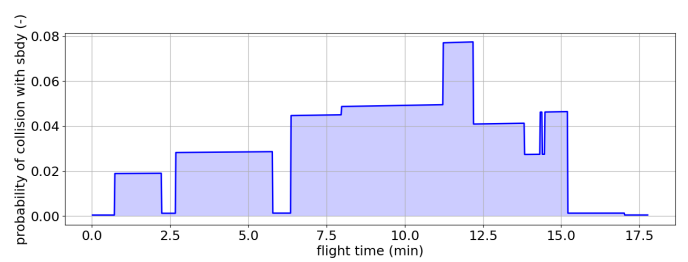

Fig. 11. Maximum probability of collision with somebody wrt flight time.

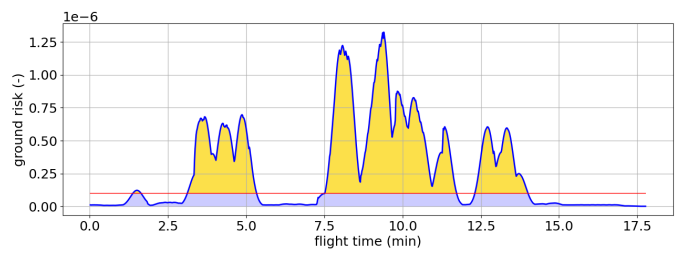

Fig. 12. Global risk probability for population wrt flight time (blue) and threshold of acceptability (red).
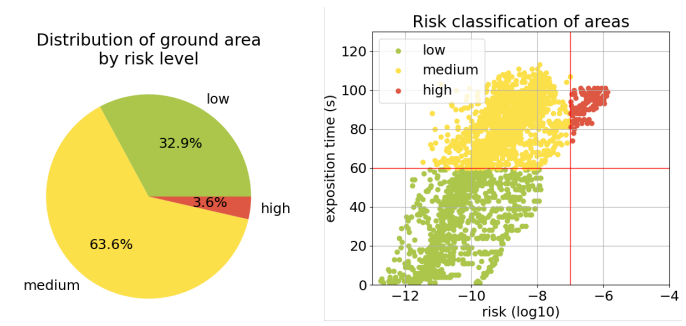

Fig. 13. Overall risk level repartition (left) and flight points'risk level repartition (right).

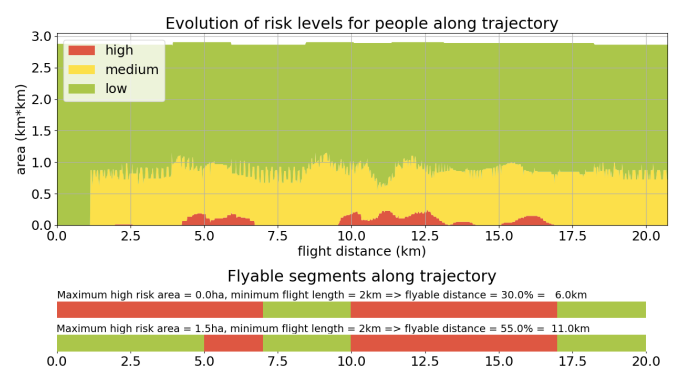

Fig. 14. Risk level for population wrt flight distance / feasible flight sections wrt to operation thresholds.

for in this process. More explanation on the definition of these bar graphs can be found in Bertrand et al. (2019) as well as an example of their use in the analysis of flight trajectories for inspection of the French railways network by UAVs.

All these data computed by DROSERA tool have been used for risk assessment of several operational flight missions and helped in the definition of some of them by identifying specific issues that were not identified in the initial mission 
preparation phase.

\section{Conclusions}

This paper has presented the DROSERA tool developed for probabilistic risk assessment of UAV missions wrt third parties. It enables automated computation of quantitative risk indicators using models regarding fixed-wing UAVs and their environment. New developments by the authors have also been presented for ground impact probability computations and models of third parties's presence. Several results from a case study corresponding to a long range flight in a semi-urban area have been proposed to illustrate the risk indexes related to the developed method and computed by the tool.

Ongoing works concern improvements of the models, their adaptation to rotary-wing UAVs, and risks regarding other types of third parties. Combination of DROSERA along with MBSA tools and stochastic simulation is also under investigation, as initiated in Serru and Delmas (2021), to assess the risk as a whole accounting accurately both for vehicle, flight procedures, mission and environment.

\section{Acknowledgement}

- Initial development of the DROSERA tool has been done in the context of the research partnership DROSOFILES on UAVs between SNCF Réseau (French national railways company), Altametris (its drone subsidiary) and ONERA.

- Ongoing work is done in the context of the research partnership ARDAM on risk assessment for UAVs between Altametris, RTE (French electricity transportation network) and ONERA.

\section{References}

Bertrand, S., N. Raballand, S. Lala, and F. Viguier (2019). Feasibility analysis of UAV operations for monitoring of infrastructure networks: A risk-based approach. In 2019 International Conference on Unmanned Aircraft Systems, pp. 1286-1295. IEEE.

Bertrand, S., N. Raballand, and F. Viguier (2018). Evaluating ground risk for road networks induced by UAV operations. In 2018 International Conference on Unmanned Aircraft Systems, pp. 168-176. IEEE.

Bertrand, S., N. Raballand, F. Viguier, and F. Muller (2017). Ground risk assessment for long-range inspection missions of railways by UAVs. In 2017 International Conference on Unmanned Aircraft Systems, pp. 1343-1351. IEEE.

Bieber, P., J.-L. Farges, X. Pucel, L.-M. Séjeau, and C. Seguin (2018). Model-based safety analysis for co-assessment of operation and system safety: application to specific operations of unmanned aircraft. In 9th European Congress Embedded Real Time Software and Systems.

Clothier, R., R. Walker, N. Fulton, and D. Campbell (2007). A casualty risk analysis for unmanned aerial system (UAS) operations over inhabited areas. In 2nd Australasian Unmanned Air Vehicles Conference, pp. 1-16.

Dalamagkidis, K., K. P. Valavanis, and L. A. Piegl (2008). Evaluating the risk of unmanned aircraft ground impacts. In 16th Mediterranean Conference on Control and Automation, pp. 709-716. IEEE.

Delmas, K., C. Seguin, and P. Bieber (2019). Tiered model-based safety assessment. In International Symposium on Model-Based Safety and Assessment.

INSEE (2012). L'emploi du temps en 2010. Enquête emploi du temps https://www.insee.fr/fr/statistiques/2118074. INSEE Résultats no130, june 2012.

Levasseur, B., S. Bertrand, and N. Raballand (2020). Efficient generation of ground impact probability maps by neural networks for risk analysis of UAV missions. In 2020 International Conference on Unmanned Aircraft Systems, pp. 1398-1406. IEEE.

Levasseur, B., S. Bertrand, N. Raballand, F. Viguier, and G. Goussu (2019). Accurate ground impact footprints and probabilistic maps for risk analysis of UAV missions. In IEEE Aerospace Conference, pp. 1-10.

Melnyk, R., D. Schrage, V. Volovoi, and H. Jimenez (2014). A third-party casualty risk model for unmanned aircraft system operations. Reliability Engineering \& System Safety 124, 105-116.

Rattanagraikanakorn,

B., D. I. Gransden, M. Schuurman, C. De Wagter, R. Happee, A. Sharpanskykh, and H. A. Blom (2020). Multibody system modelling of unmanned aircraft system collisions with the human head. International journal of crashworthiness 25(6), 689-707.

Serru, T. and K. Delmas (2021). A comprehensive probabilistic assessment method of UAS ground collision risk. In 31st European Safety and Reliability Conference.

Washington, A., R. A. Clothier, and J. Silva (2017). A review of unmanned aircraft system ground risk models. Progress in Aerospace Sciences 95, 24-44.

Wu, P. and R. Clothier (2012). The development of ground impact models for the analysis of the risks associated with unmanned aircraft operations over inhabited areas. In 11th International Probabilistic Safety Assessment and Management Conference and the 2012 Annual European Safety and Reliability Conference, pp. 5222-5235. 